\title{
Monitoring System of High Voltage Dielectric Equipment
}

\author{
Aleksandr V. Golenishchev-Kutuzov ${ }^{1}$, Vadim A. Golenishchev-Kutuzov ${ }^{1}$, Dmitry A. Ivanov ${ }^{1, *}$, Anton V. Semennikov ${ }^{1}$, and \\ Tatyana G. Galieva ${ }^{1}$
}

${ }^{1}$ Kazan State Power Engineering University, 420066, 51 Krasnoselskaya St., Kazan, Russia

\begin{abstract}
Based on the research of the main causes of dielectric elements premature aging and electrical destruction of primary dielectric elements in high voltage electrical equipment the complex method for remote monitoring of said electrical equipment has been developed. The developed mobile diagnostic device is allowing detection and periodic control of the most dangerous defects. The set of diagnostic parameters of defects was experimentally obtained by using three physical methods (electromagnetic, optical, and acoustic), and allows to inspect the origin and development of defects, and to determine residual life of dielectric elements with a sufficient degree of reliability. The researched characteristics of the kinetics of reduction in operating parameters of polymer insulators permitted the development of the detection of accelerated increase in defects under the influence of ultra-large partial discharges.
\end{abstract}

\section{Introduction}

Due to its complexity, application of various materials and operation under certain conditions with the exposure to strong electric and electromagnetic fields, electrodynamic and thermal effects, the high-voltage electrical equipment is a subject to the increased risk of defects, followed by malfunctions and failures. Therefore, the purpose of technical diagnostics is to unambiguously identify defects and predict their further development as well as the residual life of high-voltage equipment. In recent decades, has been developing a transition from the system of scheduled preventive maintenance of high-voltage equipment to the maintenance based on technical condition, including the use of non-destructive testing of the technical condition of equipment. The main difference between this and previously developed and used diagnostic methods is the ability to inspect equipment without interrupting the process of power transmission [1-10]. A quick and successful transition to the diagnostic method under operating voltage is hampered by several current problems that require advanced solutions:

- Absence of the regulatory documents that include critical diagnostic parameters, based on which a decision can be made on the technical condition; inconsistency of the parameters obtained during equipment shutdown and the parameters when using the "under operating voltage" diagnostics method.

- Lack of the methods of multiple diagnostics with a certain periodicity, such as monitoring of diagnostic parameters. In our opinion, the periodicity should be determined by the rate of possible formation and development of a defect in any controlled item of equipment.
- Non-availability of the most optimal set of measuring systems and devices for remote diagnostics or for monitoring the operation of high-voltage equipment; lack of the advanced software systems that would allow for automatic diagnostics and could provide a decision whether to decommission the equipment.

- Non-existence of universal and reliable methods for the remote non-contact diagnostics of power systems.

The proposed article partially summarizes the experience of the Kazan State Power Engineering University researchers in the development of an integrated method and a corresponding monitoring system of the most critical and most vulnerable nodes during the operation - the high-voltage insulators and the overhead power lines. At present, most power systems have switched to the use of polymer insulators (PI) for operating voltages up to $500 \mathrm{kV}$. However, older systems using porcelain insulators also exist. The focus of this article is on PI. Certain attention will be drawn to an equally widespread object of the electric power industry - the high-voltage power lines.

According to our representation based on previously obtained results, a perfect monitoring system should be able to establish the current technical condition of an object, determine the characteristics of the most dangerous defects, independently identify the most rapidly developing defects, and determine the frequency of diagnostic processes.

Since the second half of the 20th century, the main attention in international and Russian scientific articles has been drawn to partial discharges (PD) as the primary electrophysical processes that determine the operating state of high-voltage insulators (HVI). Moreover, in the last decade, it was discovered that PDs at a defect can create super-large gradients of electric fields

\footnotetext{
* Corresponding author: ivanov.da@,kgeu.ru
} 


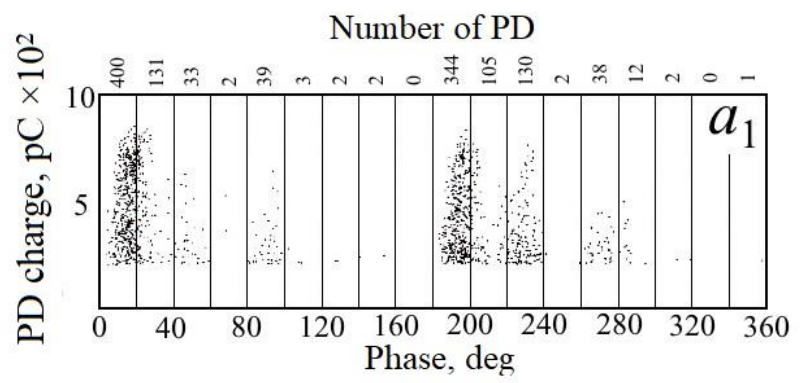

Number of PD
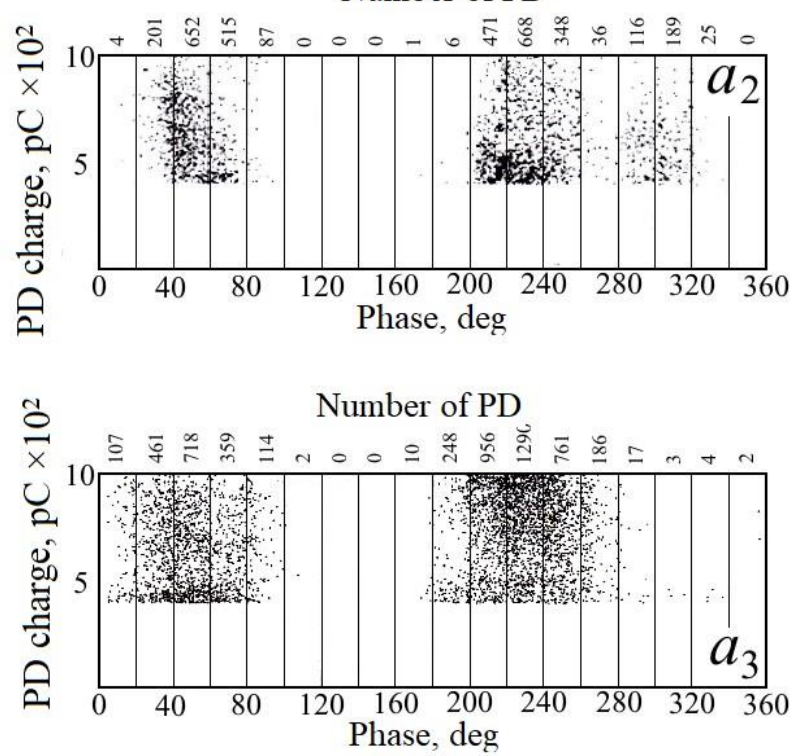

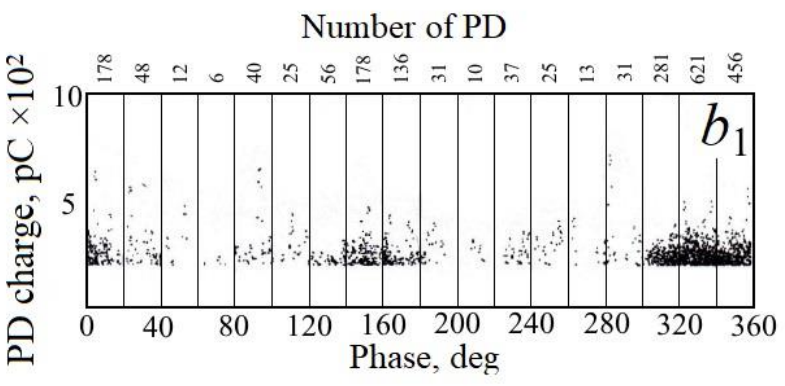

Number of PD

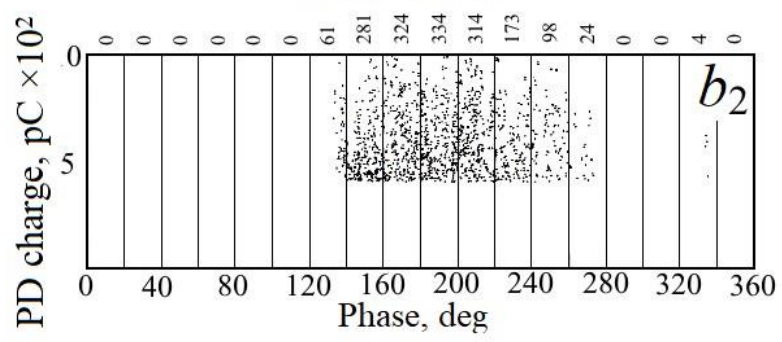

Number of PD

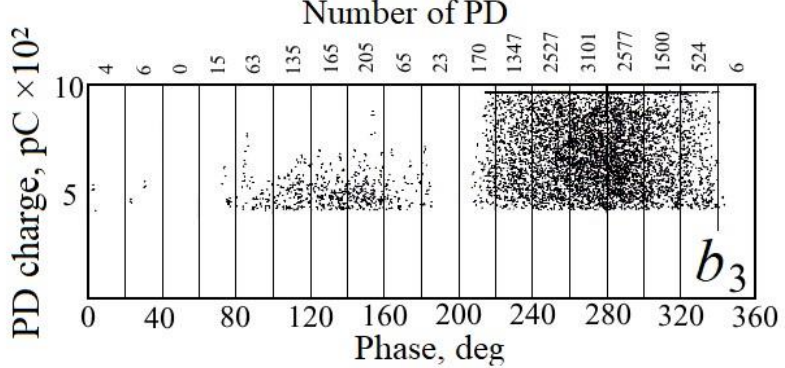

Fig. 1. Amplitude-phase diagrams of the distribution of individual partial discharges for HVI containing defects on the rod (a) and defects at the contact of "rod - electrode tip" (b): 1 - initial development of defects, 2 - further developed defects containing ultra-large PD, 3 - large defects posing a danger to further operation.

significantly exceeding the field strength in a highvoltage electrical network [17].

\section{Control of Defects Using Partial Discharges}

Three main types of PD are distinguished in HVI: microdischarges in small cavities existing both on the surfaces of electrodes and in the bulk of dielectrics; partial discharges along the interface between two dielectrics; partial (incomplete) breakdowns at the "solid dielectric gas" interface. Corresponding to them are the defects, most often in the form of spherical air cavities inside dielectrics and plane-parallel air layers inside the dielectric and at the "dielectric - electrode" interface (disk cavities). The detection of extra-large PD (extralarge PD) and the developed set of diagnostic parameters for their detection have already been reported before [1115]. However, the physical principles of the development of defects in polymeric materials under the action of extra-large PD have not been developed, and models of progressing defects and residual life based on new diagnostic parameters have not been built [16-17].
When using the already known and established methods of complex diagnostics of HVI operability [6], it is possible to determine the important diagnostic parameters of defects (such as type, location, size, development rate, degree of influence on further operability) by periodically measuring the developed set of characteristics of partial discharges, which provides values for diagnostic parameters of defects.

For further justification of the developed method, the measurements were taken of a set of corresponding characteristics of partial discharges on a series of polymer and porcelain high voltage insulators containing various types of the most dangerous defects, differing in location and size. Characteristics were measured at the stand and at the substations with the use of electromagnetic and acoustic receivers. Fig. 1 presents the amplitude-phase diagrams of the distribution of individual PDs for insulators containing defects on the rod (a) and defects on the contact "rod-electrode tip" (b). The upper diagram (1) corresponds to the initial development of defects that do not pose a danger to the operation of the insulators. Diagrams (2) refer to further developed defects and contain ultra-large PDs with a charge $\mathrm{q}>2 \mathrm{nC}$. For defects on the rod, they are located symmetrically on the positive and negative phase half- 
periods of the high voltage. For "rod - electrode tip" defects, these discharges are typical only for a negative half-period. Diagrams (3) show the PD distributions for samples with large defects, which are dangerous for further operation. They are characterized by a significant increase in the number and charge of ultra-large PDs, as well as an expansion of the generation phase intervals.

\section{The Role of Electrophysical Processes in the Formation of Defects}

The PD process on the surface or in the bulk of the dielectric can be conventionally divided into two periods. In the first period, there is ionization of air or a gas that fills the cavity of the defect. Under the action of the applied field, originating the free electrons, positive and negative ions, which, depending on the charge, circulate in the cavity and bombard the dielectric cavity of the defect. Previously, often studied were the processes of generation and spread of PD in the form of short electric pulses with a duration of $10^{-7}$ seconds to $10^{-11}-10^{-12}$ seconds in the defect cavity $[1,7,8]$. The influence of PD on the dielectric strength was also researched. In recent years, the attention of researchers and practitioners has switched to studying the processes of PD formation with increased intensity (ultra-large PD) due to the accumulation of electric charges from previous PDs on the surfaces of defects. The second period is longer lasting and ends with the redistribution of charges on the defect surfaces and their recombination. In this case, the field inside the cavity of the defect takes on the value:

$$
E_{C}=E_{B}+E_{a},
$$

where $E_{B}=\frac{q}{g \varepsilon_{0}}-$ field generated by induced charges on the defect surface; $E_{a}$ - applied field to the insulator. Stream-type PD processes have been well studied by now $[6,7,17]$. Therefore, the main attention was paid to the influence of charged defect surfaces on the parameters of subsequent PDs. Until now, this study has been carried out mainly on model samples of polymer dielectrics containing defects in the form of spherical cavities $[9-11,14,16]$. We researched the features of various defects in polymer and porcelain HVIs for the first time. In particular, it was found that extra-large PDs appear on the rod and the contact of "rod - electrode tip" under practically the same conditions. The differences begin only in the second period - the propagation of surface charges on the surfaces of defects.

\section{Diagnostic Parameters for Detection of Extra-Large PD}

Based on the results of examination of series of polymer (LK35 / 70) and porcelain (IOS 110/400) insulators by using the contact and remote methods [5], the most important diagnostic parameters were established, which largely complement the similar parameters recommended by GOST R55191-2012.

These parameters include the presence of ultra-large PDs, their phase distribution, phase displacement, and an increase in charge over time. The presence of the most significant defects is determined by the occurrence of ultra-large PD. The highest limit values are determined by the material and type of high-voltage insulators.

The type and location of the most serious defects are determined by the phase distribution of ultra-large PD. The defects on the shell of the insulator rod are characterized by their symmetrical arrangement in positive and negative half-periods of the applied voltage. The presence of super-large PDs with only a negative half-period corresponds to a defect on the contact of "rod - electrode tip".

The rate of development of the most critical defects is determined by the change in diagnostic parameters such as shift of ultra-large PDs into phase intervals with the approach to the beginning of half periods $\left(0^{\circ}\right.$ and $180^{\circ}$ ), as well as increase in the apparent charge in the time interval between the two consecutive measurements of diagnostic parameters. The rate of development of the most critical defects is used to predict the residual life of the insulators operative condition.

The method of remote monitoring of the technical condition of high-voltage insulators is carried out through the following operations:

- At the first stage, for each type of high-voltage insulators, the limiting values of the parameters of the characteristics of extra-large PD are established by using the contact method recommended by GOST; these limiting values do not create breakdown of insulator defects but affect the rate of development of defects.

- During the next step, these normalized diagnostic parameters established by the contact method are compared to the values of the same partial discharge parameters measured remotely by a radio frequency receiver with a narrow beam antenna. Then, the pulsed signals of partial discharges, synchronized with the high voltage phase and accumulated for at least $18 \mathrm{~s}$, are distributed in accordance with charge, quantity and phase angles established by the developed computer program; and form the necessary set of diagnostic parameters to characterize the condition of the insulator at the time of this measurement. The processed parameter set is then saved to the database.

- At the last stage, the conclusion is made about the residual resource (service life) of the diagnosed insulators, as well as the frequency of further remote measurements by comparing the results of the current measurement of diagnostic parameters values with the values of the same parameters obtained during the previous remote measurements.

\section{Development of the Concept of a System for Diagnostics of High-Voltage Dielectric Elements During Operation}

Receiving antennas of various shapes are used to measure $\mathrm{PD}$ in the ultrahigh frequency range; then, by 
using mathematical methods, the location of the PD event is triangulated in 3 dimensions.

To detect PD in HVI, it is proposed to use noncontact diagnostic devices, the block diagram of which is shown in Figure 2. The diagnostic device takes measurements by using an electromagnetic (EM) sensor and a phase sensor. The diagnostic device is installed on the phase wire at a predetermined distance from the wire suspension point. The EM sensor uses an antenna to measure the electromagnetic field emitted by the PD.

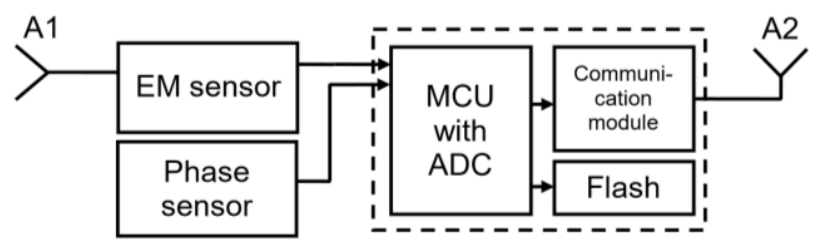

Fig. 2. Block diagram of a non-contact diagnostic device: A1 antenna of EM sensor, A2 - antenna for data transmission, ADC - analog-to-digital converter, MCU - microcontroller.

For the measurement of high frequency PD pulses, frequencies from 100 to $2000 \mathrm{MHz}$ are considered. After digital processing in the $\mathrm{ADC}$, the received data from the two sensors are processed in the microcontroller and transferred to the storage device.

In this case, the complex consists of three diagnostic devices, one device per phase wire. It is a receiving antenna array (Fig. 3). The difference in time of arrival (TDOA) between the received signals at each of the corresponding diagnostic devices is determined, which makes it possible to deduce the three-dimensional position of the electric discharge source by processing the TDOA values. The said method is used in this work. Three non-contact diagnostic devices are installed on each phase wire $\left(D_{1}-D_{3}\right)$ at a certain known distance from the HVI - $r_{i}$, where $i$ is the serial number of the sensor $(i=1-3)$. The sensor operates on power lines from $10 \mathrm{kV}$.

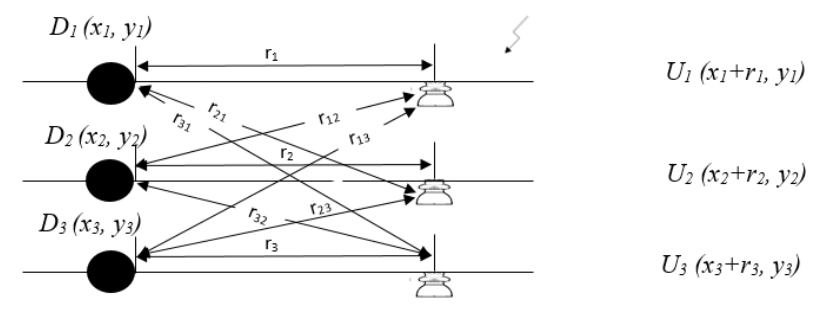

Fig. 3. Installation diagram of the diagnostic devices.

The time difference localization method consists of two main stages - the estimation of the time difference of arrival and the solution of the nonlinear equations of the time difference [18]. This method is often used for local positioning of mobile devices. The method is based on measuring the difference in the transmission time of the signal from the PD to the diagnostic devices, with time synchronization and a predetermined location. Knowing the difference in time of receiving the signal, the distance from the PD source to the diagnostic device is calculated using mathematical processes.
Knowing the coordinates of each diagnostic device installed on the phase conductor (Fig. 3) allows for calculation of the propagation time from the source of partial discharges to the corresponding diagnostic devices using the basic formula $D=v \bullet t$, where $D$ is the distance, $v$ is the propagation velocity, and $t$ propagation time. This method is described by:

$$
\left(x-x_{i}\right)^{2}+\left(y-y_{i}\right)^{2}+\left(z-z_{i}\right)^{2}=\left(V_{e} \cdot t_{i}\right)^{2}
$$

where $\left(x_{i}, y_{i}, z_{i}\right)$ are the coordinates of the $i$-th diagnostic device in Cartesian space, $(x, y, z)$ are the true coordinates of the partial discharge event, $V_{e}$ is the velocity of the electromagnetic wave, $t_{i}$ is the "time of flight" of the propagating PD signal from its source to the $i$-th sensor.

The position of the PD source $(x, y)$ can be calculated using the least square method:

$$
S(X)=\sum_{i=1}^{N}\left(Y_{i}(X)\right)^{2}
$$

After the number of PDs, their intensity and location are determined, the resulting distribution is compared with the distribution for serviceable, pre-defective and defective insulators. The aging rate and the development speed for various defects are determined by using the developed technique which makes it possible to predict the residual life of the high-voltage insulator.

\section{Conclusion}

Thus, the performed bench tests and field tests of the developed methodology and measuring complex [6, 12] showed the real possibility of its use for monitoring the state of HVIs in operation.

A concept for construction of a continuous monitoring system for the technical condition of highvoltage insulation on overhead power lines and substations has been developed.

The work was supported by the Ministry of Science and Higher Education of the Russian Federation on fundamental scientific research «Distributed automated systems for monitoring and diagnostic the technical condition of overhead power lines and substations based on technology of broadband data transmission through power lines and the Industrial Internet of Things» (theme number 075-00063-20-02).

\section{References}

1. V.Ya. Ushakov, Isolyatsiya ustanovok vysokogo napryazheniya (Insulation in High-Voltage Facilities) (Moscow: Energoatomizdat, 496 (1994)

2. L. Niemeyer, IEEE Trans. Dielectr. Electr. Insul., 2, 510 (1995)

3. Yu.N. Vershinin, Electronno-teplovye $i$ distantsionnye protsessy pri elektricheskom proboe tverdykh dielektrikov (Electronic-Thermal and Remote Processes in the Electrical 
Breakdown of Solid Dielectrics) (Yekaterinburg: Ural Branch, Russ. Acad. Sci., 260, 2000)

4. K. Wu, Y. Suzuoki, L.A. Dissado, J. Phys. D., 37, 1815 (2004)

5. A.L. Kupershtokh, C.P. Stamatelatos, D.P. Agoris, Techn. Phys. Let., 32, 8 (2006)

6. R. Bartnicas, IEEE Transactions on Dielect. and Elect. Insulation, 9, 763-808 (2002)

7. V.P. Vdoviko, Chastichnye razryady $v$ diagnostirovanii vysokovol'tnogo oborudovaniya (Partial Discharge in the Diagnosis of HighVoltage Equipment) (Novosibirsk: Science, 156, 2007)

8. A.S. Gaivoronsky, Chief Power Engineer, 2, 2327 (2010)

9. C. Pan, Y. Meng, K. Wu, Z. Han, K. Qin, Y. Cheng, J. of Phys. D Appl. Phys., 44, 255201255208 (2011)

10. H.A. Illias, G. Chen, P.L. Lewin, J. of Phys. D. Appl. Phys., 44, 245202-245216 (2011)

11. K. Wu, C. Pan, Y. Meng, Y. Cheng, IEEE Transactions on Dielect. and Elect. Insulation, 20, 612-619 (2013)

12. A.V. Golenishchev-Kutuzov, V.A. GolenishchevKutuzov, R.A. Khusnutdinov, G.D. Mardanov, Electrotekhnika, 3, $72-73$ (2017)

13. A.V. Golenishchev-Kutuzov, V.A. GolenishchevKutuzov, G.D. Mardanov, R.A. Khusnutdinov, I.A. Evdokimov, Russian J. of Nondestructive Testing, 52 (8), 478-483 (2016)

14. A.L. Kupershtokh, D.I. Karpov, J. of Phys.: Conf. Ser., 754, 102006-102011 (2016)

15. A.V. Golenishchev-Kutuzov, V.A. GolenishchevKutuzov, D.A. Ivanov, G.D. Mardanov, A.V. Semennikov, L.V. Ahmetvaleeva, E3S Web of Conferences, 124, 03001 (2019)

16. G. Callender, I.O. Golosnoy, P. Rapisarda, P.L. Lewin, J. of Phys. D Appl. Phys., 51, 125601125624 (2018)

17. A.V. Golenishchev-Kutuzov, V.A. GolenishchevKutuzov, D.A. Ivanov, G.D. Mardanov, A.V. Semennikov, The Method of Non-Contact Remote Diagnostics of High-Voltage Insulators, Russian Fed. Patent for invention, 2679759 (2018)

18. D.A. Ivanov, T.G. Galieva, M.F. Sadykov, A.V. Golenischev-Kutuzov, A.A. Naumov, E3S Web of Conferences, 216, 01061 (2020). 